\title{
Study of CFRP adhesive bonds influenced by factors encountered during aircraft operations
}

Paweł H. Malinowski ${ }^{1 *}$, Tomasz Wandowski ${ }^{1}$, Wiesław M. Ostachowicz ${ }^{1}$, Angelos Christopoulos ${ }^{2}$, Ilias Koulalis ${ }^{2}$, Konstantinos Kitsianos ${ }^{2}$, George Kanterakis ${ }^{2}$, Romain Ecault ${ }^{3}$, Rainer Stoessel ${ }^{4}$, Damien Segur ${ }^{5}$, Laurent Berthe ${ }^{6}$, Maxime Sagnard ${ }^{6}$, Fabienne Touchard $^{7}$, Michel Boustie ${ }^{7}$, Welchy Leite Cavalcanti ${ }^{8}$, Kai Brune ${ }^{8}$

${ }^{1}$ Institute of Fluid-Flow Machinery, Polish Academy of Sciences

Fiszera 14, 80-231, Gdansk, Poland

${ }^{2}$ GMI Aero SAS, 9 rue Buffault, Paris, 75009, France

${ }^{3}$ Airbus Operations S.A.S, 316 route de Bayonne B.P. D4101, 31060 Toulouse Cedex 9, France

${ }^{4}$ Airbus Group Innovations, Willy-Messerschmitt-Strasse 1, 82024 Taufkirchen, Germany

${ }^{5}$ CEA Tech Occitanie, 135, avenue de Rangueil | INSA Bât. 17 | 31400 Toulouse, France

${ }^{6}$ PIMM, CNRS-ENSAM Paristech, 151 Bd de l'Hopital, 75013 Paris Cedex, France

${ }^{7}$ Institut PPRIME, CNRS, ISAE-ENSMA, Université de Poitiers, 86961 Futuroscope-Chasseneuil, France

${ }^{8}$ Fraunhofer IFAM, Adhesive Bonding Technology and Surfaces,

12 Wiener Street, Bremen, 28359, Germany

Keywords: composites, CFRP, NDT, NDE, contamination, adhesive bonds, weak bonds

\begin{abstract}
Composite materials are commonly used in many branches of industry. One of the effective meth ods to join CFRP parts is to use adhesives. There is a search of effective methods for quality assurance of bonded parts. There is a need for pre- and post-bond inspection to ensure proper bonding and verify its quality. Research reported here focuses on post-bond inspection of bonded CFRP parts. In this paper we report investigations of samples that were modified in order to simulate the conditions that can be encountered during the bonding repair processes. The modifications were made before adhesive bonding, and their effect on the quality of the bond is studied. The first case was the thermal treatment. It was made by exposure of samples to elevated temperatures. This case accounts for parts that may be exposed to external heat source or lightening impact. Second of the investigated cases was deicing fluid contamination prepared by dip-coating of clean CFRP plates. When cleaning the aircraft for a repair this fluid can be transported to bonding areas and weaken the joint. The third type of modification was faulty curing of the adhesive. It was prepared by local pre-curing of the adhesive. Pre-curing causes irregularities in the curing of the adhesive joint. Apart from single modifications, also mixed cases were studied, as well as scarf bonding. The samples were studied in non-destructive approach. It was shown that for some of the cases the detection is possible.
\end{abstract}

*pmalinowski@imp.gda.pl; phone +48 585225 174; fax +48 5834161 44; www.imp.gda.pl/en/o4/z1/ 


\section{INTRODUCTION}

In recent years there is great interest observed in replacing traditional joints by adhesive bonding. This joining method is thought as the most optimal for fiber reinforced polymer composites. There is a search of effective methods for quality assurance of bonded parts. There is a need for pre- and post-bond inspection to ensure proper bonding and verify its quality. Research reported here focuses on post-bond inspection of bonded CFRP parts. In this paper we report investigations of samples that were modified in order to simulate the conditions that can be encountered during the bonding repair processes. Three types of modifications were considered. The first modification was the thermal treatment before bonding. The second case was prepared by pre-bond contamination of the sample with de-icing fluid. The third type of modification was more local - the adhesive on the sample surface was locally cured by heating before the proper bonding procedure was started. Apart from the single modifications, also mixed cases was studied. The samples after thermal treatment were contaminated with the de-icer. The pre-bond contamination or modification have an influence on the fracture toughness of the CFRP bonded joints. According to the literature, the pre-bond thermal treatment results in decrease of GIC value by 10 and $13 \%$ for treatment at 190 and $200^{\circ} \mathrm{C}$, respectively [1]. In the case of treatment at $210^{\circ} \mathrm{C}$ the increase by $9 \%$ was observed [1]. In the case of improper curing of the adhesive, it was shown that the lowered temperature have great impact on the bond performance. The GIC for sample cured at $120^{\circ} \mathrm{C}$ drops nearly by $95 \%$ in relation to GIC for sample properly cured at $180^{\circ} \mathrm{C}$ [2]. As far as the de-icing fluid is considered it was shown that it has a negative effect on both mode I and mode II fracture toughness of the bonded joints. The increasing level of contamination results in decrease of the mode I and mode II critical energy release rates [3]. In this paper we report results of five techniques employed for assessment of the bonds. Micro computed tomography, non-linear ultrasound, magnetostrictive sensors, laser shock adhesion test, and the electromechanical impedance (EMI) technique were investigated. The micro computed tomography was used as a referential method giving information about the pore content in the bond. The non-linear solitary was were used before for adhesive bond with release agent contamination [4]. Magnetostrictive sensors were used as strain sensors. Changes of strain were investigated for assessing the adhesive bonding. In past the strain gradient were investigated by embedding fibre Bragg grating (FBG) sensors in the samples [5]. The EMI method was investigated before for this cases but only for single modifications [6]. In the next section the samples are described together with contaminations types and their possible origin in real parts. The third section presents the five investigated methods. The fourth section contains results for each of the technique. The paper ends with conclusions regarding each technique.

\section{INVESTIGATED SAMPLES}

The material used for samples was Hexcel M21E. The size of samples was $10 \mathrm{~cm}$ x $10 \mathrm{~cm}$. The samples comprised of two plates adhesively bonded together with FM300-2 film adhesive. Each plate was made with 8 plies and layup sequence: $[0,0,45,-45] \mathrm{s}$. The adhesive was cured at $121{ }^{\circ} \mathrm{C}$. Reference samples (properly bonded) and samples with bond modification were prepared. The reference samples were marked as RRE.

Structural parts that may be exposed to external heat source, lightening impact, hot engine exhaust gases. High temperatures can cause local overheating and damage the resin. It leads to a loss of the mechanical properties of the CFRP structure and may affect the bonded repair of the thermally treated area. The thermal treatment was performed by putting the samples in an oven with air circulation. Three temperature levels were used: 220 (RTD1), 260 (RTD2) and $280^{\circ} \mathrm{C}$ (RTD3). Samples where kept for $2 \mathrm{~h}$ in these chosen thermal conditions. After the exposure to elevated temperatures, the samples were grinded before bonding. Three samples were prepared for each temperature.

The pre-bond contamination case was prepared by dip coating of the plates in a water solution of the SAFEWAY KF deicer with three concentrations. This runway de-icing fluid that can affect the aircraft parts. After drying the potassium formate stays on the CFRP in a form of a thin layer. When cleaning the aircraft, this substance can be transported to areas being prepared for repair bonding and contaminate them. This may result in a weak adhesive bond. After the initial studies it was decided to prepare samples in solutions with the following concentrations: $2 \%, 7 \%$ and $10 \%$ of de-icer in demineralized water. With the same de-icer concentrations, The dip coating results were controlled by XPS (X-ray photoelectron spectroscopy) measurements. Since the de-icer contains potassium formate, the potassium (K) content on the surface is taken as a measure of the degree of contamination. For the considered concentrations following 
contamination levels were obtained: $6.4 \pm 1.8$ (RDI1), 10.9 \pm 2.3 (RDI2) and 12.0 \pm 1.4 at.\% K (RDI3). The initial lap shear tests showed a significant loss in bond strength for approximately 4 at $\% \mathrm{~K}$. Three samples at each level were prepared. The case of faulty curing of adhesive (sample symbol: RFC) was prepared by local pre-curing of the adhesive by an infrared source. This pre-curing was made at the middle of the sample. The simulated loss of performance of the bonded joint is related to irregularities in the curing of the adhesive that was caused by pre-curing. The adequate adhesive bonding processes requires that all parameters like curing times, shelf life, etc. comply will the regulations. Such precuring could happen accidentally during the repair process.

The mixed modification case comprised of thermal treatment at the level 1 combined with de-icer contamination at level 1 (DI1) and level2 (RDI2). The same mixed modifications were used for the samples with scarfed bonding. The samples with scarfed bonding were a bit different in size than the previously described samples. Two plates with a size of $12 \mathrm{~cm}$ x $12 \mathrm{~cm}$ were bonded with an overlap- scarfed area of about $6 \mathrm{~cm} \times 12 \mathrm{~cm}$.

\section{BONDS ASSESSMENT METHODS}

In the frame of the reported research five methods were investigated for the assessment of the adhesive bonds: micro computed tomography, non-linear ultrasound, magnetostrictive sensors, laser shock adhesion test, and the electromechanical impedance technique.

\subsection{Micro-Computed Tomography}

The micro-computed Tomography ( $\mu$-CT) was used to evaluate the degree of adhesive layer porosity at the bond line and gathering 3D information. The CT testing the system shown in Figure 1a) was used. The investigated sample is placed on the turn-table (Figure 1a) for obtaining the x-ray images. Based on the images the 3D data are evaluated Figure 1b). The samples were tested in a form of stacks of three samples within on measurement. Since mainly the centre section of the samples should be tested a region-of-interest of about $60 \mathrm{~mm}$ x $60 \mathrm{~mm}$ was defined. The voxel grid size was $62 \mu \mathrm{m} \times$ $62 \mu \mathrm{m} \times 62 \mu \mathrm{m}$. To reduce the image noise, three images at one angle were averaged and the number of projections was 1600. An example of the 3D data obtained is displayed in Figure 1b).

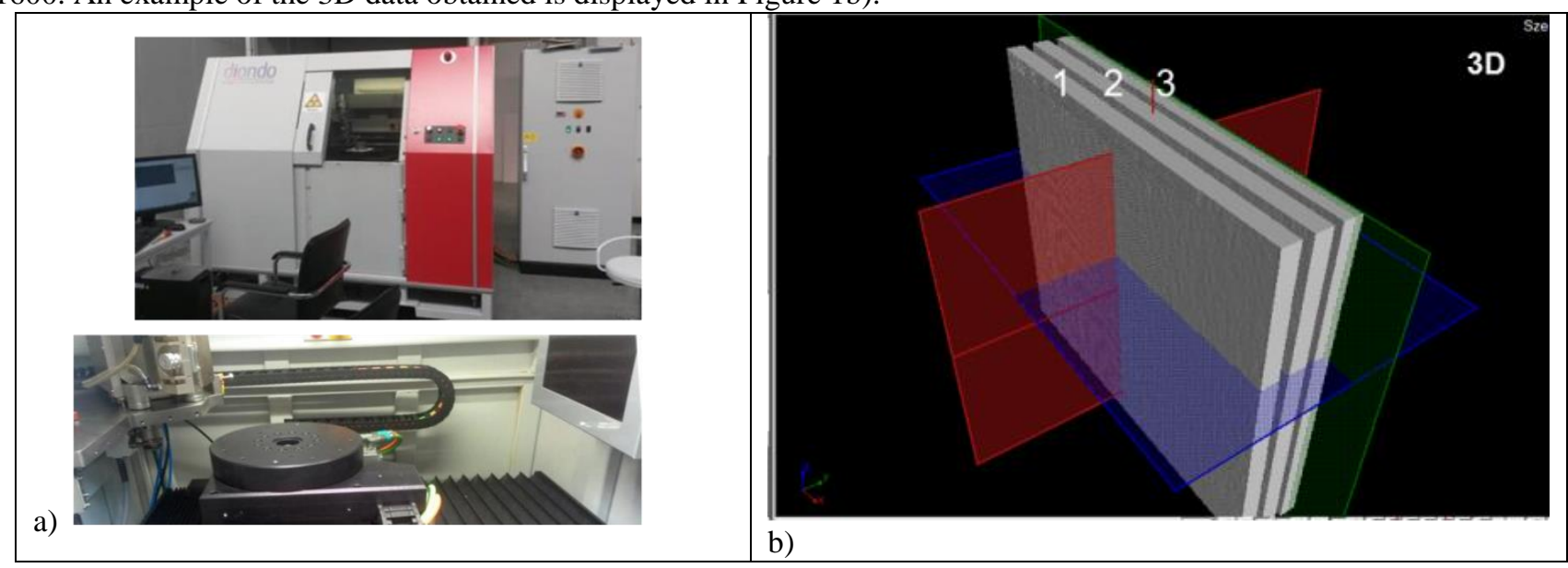

Figure 1. a) $\mu$-Computed Tomography System for CFRP; b) 3D image showing the three cutting planes

The evaluation of the porosity degree was mainly based on front cutting plane images. Due to the thickness of the sample only the layer porosity could be calculated. The layer of porosity was calculated as the sum of pores area in relation to the total investigated area of the sample.

\subsection{Non-linear ultrasounds}

In the non-linear approach the distortion of the driving excitation is detected and analyzed by using scanning laser vibrometer with bandwidth of $1.5 \mathrm{MHz}$. The amplitudes of the higher harmonics $(\mathrm{HH})$ are recorded. The quadratic-sum of the $\mathrm{HH}$ intensities is employed as a parameter of nonlinearity and used for quantifying and comparing bonding quality. In order to achieve higher amplitude of excitation piezo-actuators with a frequency response extended up to 200 
$\mathrm{kHz}$ were employed. The actuators are vacuum attached to the specimens. The actuator is attached to one of the sides of the specimen and nonlinear vibrations are measured on the opposite side by scanning laser vibrometer. The dynamic range of the vibrometer measurements is $100-120 \mathrm{~dB}$ which is well beyond the level of nonlinear frequency components. To avoid an impact of reflections on the local vibration in the excitation area, the edges of specimens were covered with dissipative material and $49 \mathrm{kHz}$ vibration frequency was chosen. After Fast Fourier Transform, maximum values of the fundamental frequency vibration velocity $\left(v_{0}\right)$ and the $\mathrm{HH}$ components $\left(v_{n}\right)$ in the excitation area are measured and used for evaluation of the specimen nonlinearity. The amplitudes of the vibration velocities is recorded and squared to constitute vibration energy spectrum. A ratio $(N)$ of nonlinear vibration energy $\sim \Sigma_{n} v_{n}^{2}$ to the energy of the fundamental frequency vibration $\sim_{0}^{2}$ is used as a measure of nonlinearity.

\subsection{Magnetostrictive strain sensing}

In this technique the strain is sensed using the magnetostrictive properties of the sensing patch. Magnetostrictive ribbons are used. In such materials the magnetic permeability is highly dependent on external mechanical loading (Villari effect). For this application the ribbons are placed in parallel and they are combined with an adhesive film (3M/AF-163-2k) forming a strain sensitive patch. After the bonding of the sensing patch the inspection of the sample is performed using the prototype inspection system that has been developed by GMI Aero (Figure 2). The data acquisition system is connected to the inductive transducer and laptop. The data acquisition system records the signal. On top of the inductive transducer an infrared LED is attached in order to track the position of the transducer wit IR camera. The camera is connected to the computer through a Bluetooth device providing the data to the sensing software.

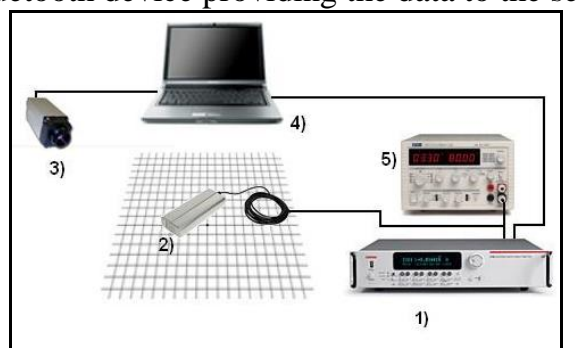

Figure 2. The schematic representation of the inspection system

\subsection{Laser shock adhesion test (LASAT)}

The LASAT technique sollicitates interfaces by shock wave produced by plasma-laser [7]. Laser power density is typically in the range of 1-5 GW/cm2, pulse duration 8-10 ns and spot diameter $8 \mathrm{~mm}$. In this application, the interaction is confined using a water layer, allowing a higher pressure to be generated than without the water layer [8]. Presently, two laser beams are impacting the sample from both sides(Figure 3). With this symmetrical setup, three tensile stress location are created when both the release waves meet at bond interface (blue circle) and at skin depth of two plates (black circle). Preliminary tests have showed that only bond interfaces (blue circle) are delaminated in the range of laser power density reached for this application. The benefit is that stresses is focused at the bond location in playing with the delay between the two laser beams.

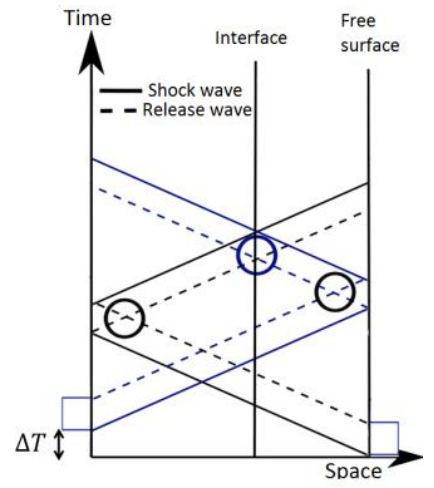

Figure 3. Space-time diagram of the LASAT technique with symmetrical set-up 


\subsection{Electromechanical impedance (EMI)}

In the electromechanical impedance (EMI) technique electric quantities of a piezoelectric sensor are measured. This sensor was bonded on the surface of the investigated samples or can be embedded inside the material. Due to relatively small samples sizes only one sensor per sample was used. The piezoelectric sensor serves for excitation and and sensing of the response. The electromechanical coupling causes that the registered impedance spectra are modified by the presence of the host structure and it's mechanical conditions (presnece of damage). additional resonance peaks, peak shiftS or magnitude change can be treated as indicator of change (damage, debonding, etc.) within the object. In order to extract damage related features for the EMI spectra various frequency bands are analysed. In the reported research we limited ourselves to $5 \mathrm{MHz}$ because this was the maximal frequency possible for the used equipment: HIOKI Impedance Analyzer IM3570.

\section{RESULTS}

\subsection{Micro-computed Tomography}

The bond lines of the three reference samples contain porosity below the evaluation limits. Therefore, no degree of layer porosity could be evaluated (Figure 4). The CT images for the thermal degradation samples RTD1 (low intensity) reveal the porosity at the level of $0.7( \pm 0.5) \%$. The values for RTD2 (medium) and RTD3 (high) are $0.7( \pm 0.2) \%$ and 0.6 $( \pm 0.3) \%$, respectively. The average degree of layer porosity of RDI samples are $1.0( \pm 0.6) \%, 0.6( \pm 0.1) \%$ and 0.3 $( \pm 0.1) \%$ counting for the lowest to the highest degree of contamination.

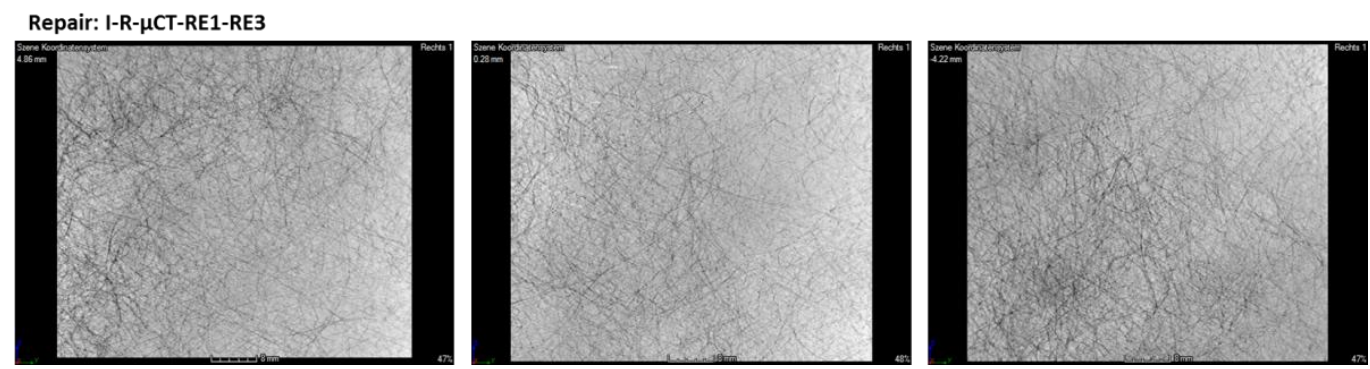

Figure 4. CT resulting images: Reference samples for repair case (RRE1-RRE3)

Considerable high degree of porosity is seen for RFC1 samples (Figure 5). The porosity ranges from $3.9 \%$ to $4.3 \%$ with an average of $4.0 \%$ and standard deviation of $0.3 \%$.
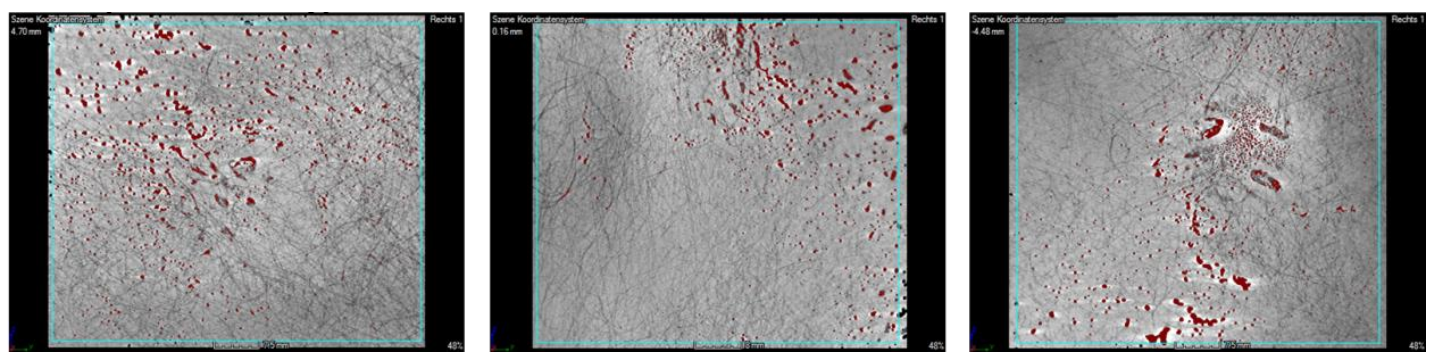

Figure 5. CT resulting images: Faulty cured samples at level 1 (RFC11-RFC13)

Next, the CT testing was first performed on the reference samples with scarfed bonding. On the images the slope of the adhesive bonding and the porosity at the area of the bonding are displayed. All three reference samples show a much higher degree of porosity in comparison to flat samples. Among the reference repair samples no differences could be detected (Figure 6Błąd! Nie można odnaleźć źródła odwolania.). One of the mixed contamination cases is thermal degradation and de-icing (both at level 1). The resulting CT evaluations show again porosity within the bond line and the pores are clustered. All three samples are very similar to each other. At the next set of samples the grade of thermal 
degradation remains 1 and the de-icing contamination level. The indications do not differ in comparison with the level 1 sample (TD1DI1).
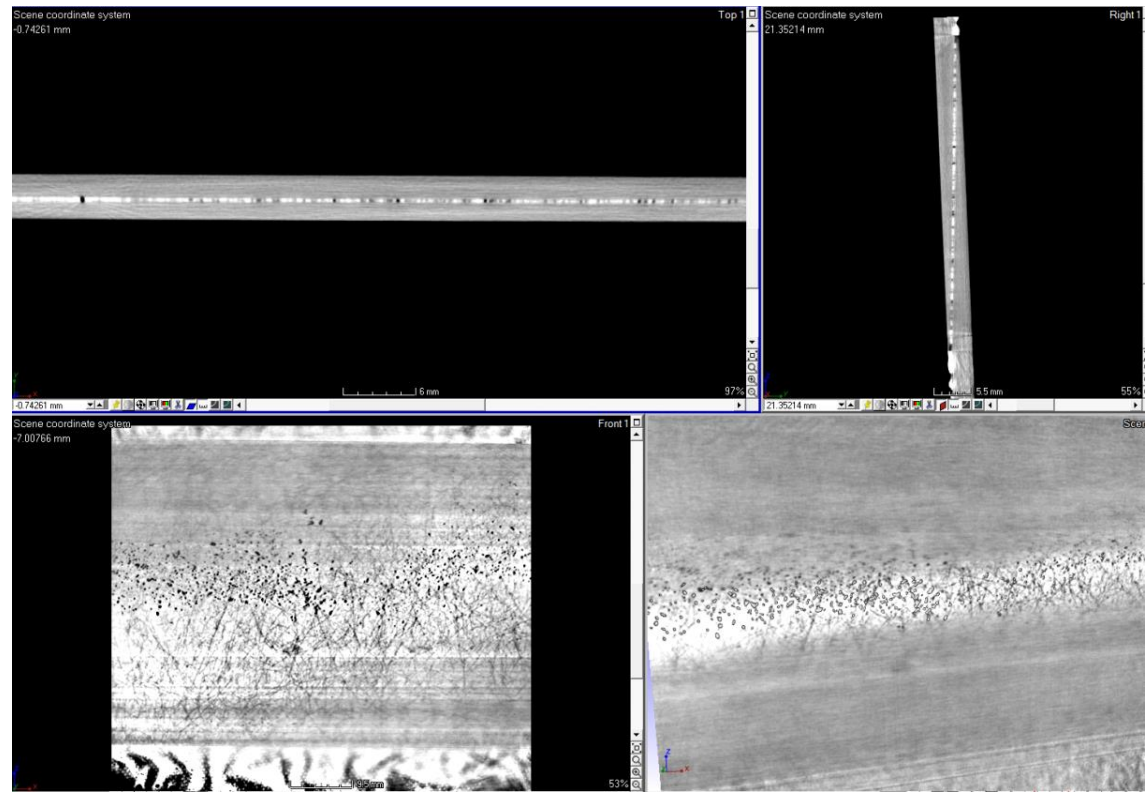

Figure 6. CT resulting images of reference sample with scarfed bonding ((upper left: cut from top; upper right: cut from right/side; lower left: cut from front; lower right: 3D display))

\subsection{Non-linear ultrasounds}

In Figure 7, the nonlinear ratio is shown that was measured on single and multiple contaminated samples. The amplitude and error bars correspond to the mean and standard deviation of data measured on the three set of samples. First, we observe that the nonlinearity ratio measured on the references samples show a minimal nonlinearity. Then, we observe a general increase of the nonlinearity with the level of contamination for TD, DI, FC and TDDI samples

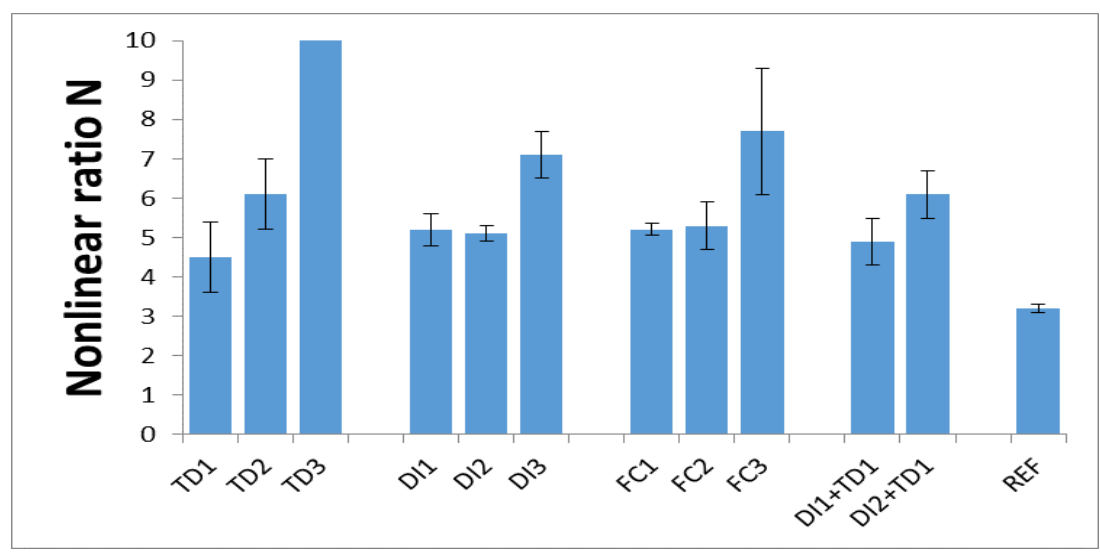

Figure 7. Measured values of ultrasonic nonlinear ratio $\mathrm{N}$ for the considered samples.

The results for scarfed samples are depicted in Figure 8. The amplitude and error bars correspond to the mean and standard deviation of data measured at five positions of the transducer on the plate. First, we observe that the nonlinearity ratio measured on the references sample show a minimal nonlinearity Then, we observe a general increase of the nonlinearity and the level of contamination for the 3 sets of samples TD+DI. 


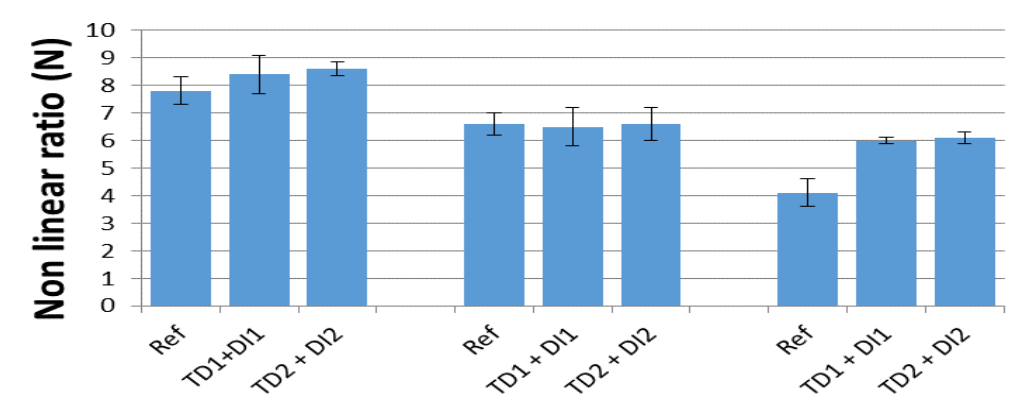

Figure 8. Measured values of nonlinear ratio $\mathrm{N}$ for contaminated specimens of scarfed samples.

\subsection{Magnetostrictive strain sensing}

The measurements were extracted using the prototype inspection system, in a region close to the centre of the test samples. Initially, the inspection is performed when the test coupon is free and no load is applied to it. Then the test coupon is subjected to four-point bending and inspected again. The data from the two states (loaded - unloaded) are then compared, by subtracting the measured values from the sensing patch. Since no clear visual evidence was possible, a statistical analysis of the extracted data was then performed, in order to increase the level of reliability of conclusions. For each type of contamination the average value and the standard deviation were calculated and compared. the average value gives us an estimation of the repeatability and the general performance of this method, while the standard deviation provides information concerning the uniformity of the strain field. a uniform strain field indicates a healthy state, while a non-uniform strain field indicates potential underling damage. The results for single modifications were presented in Figure 9, while for mixed contamination in Figure 10.

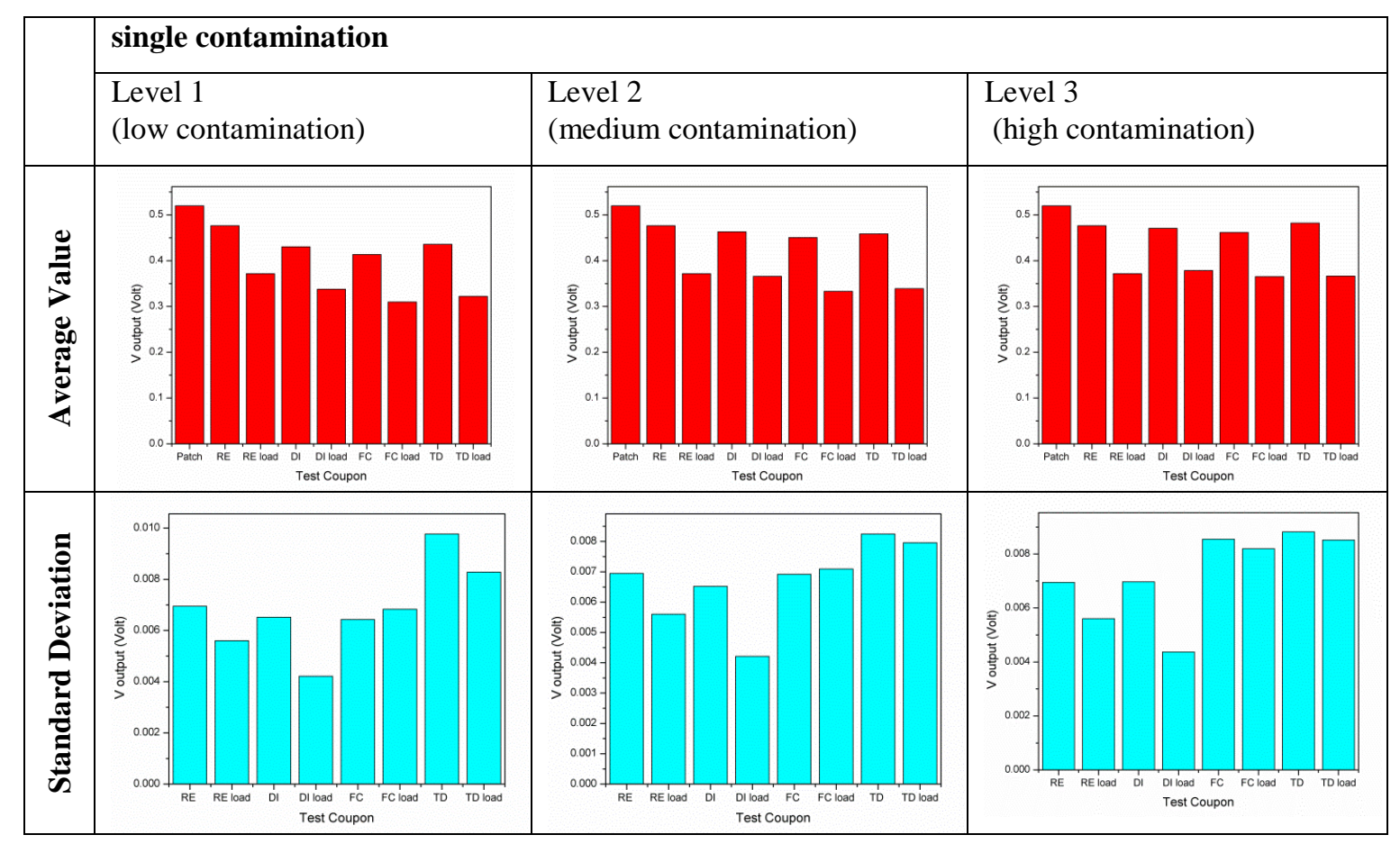

Figure 9. The average value and the standard deviation of the measurements obtained for samples with single modifications 


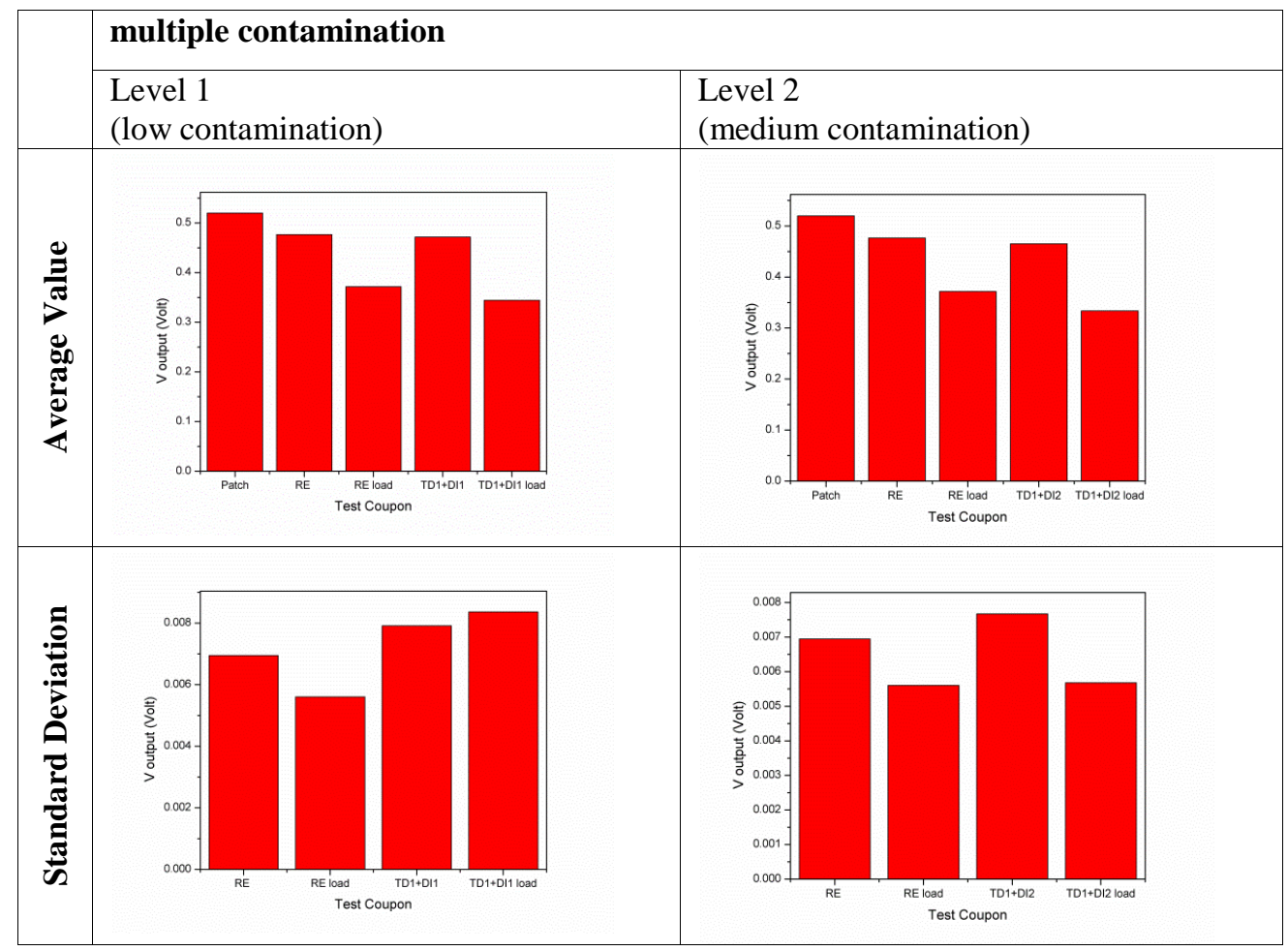

Figure 10. The average value and the standard deviation of the measurements obtained for samples with mixed modifications

The measurements for the scarfed samples were extracted using the prototype inspection system, in a region close to the centre of the scarfed area. Initially, the inspection is performed when the test coupon is free and no load is applied to it. Then the test coupon is subjected to four-point bending and inspected again. The data from the two states (loaded unloaded) are then compared, by subtracting the measured values from the sensing patch. Figure 11 illustrates characteristic examples from all types of contamination from the repair pilot samples.

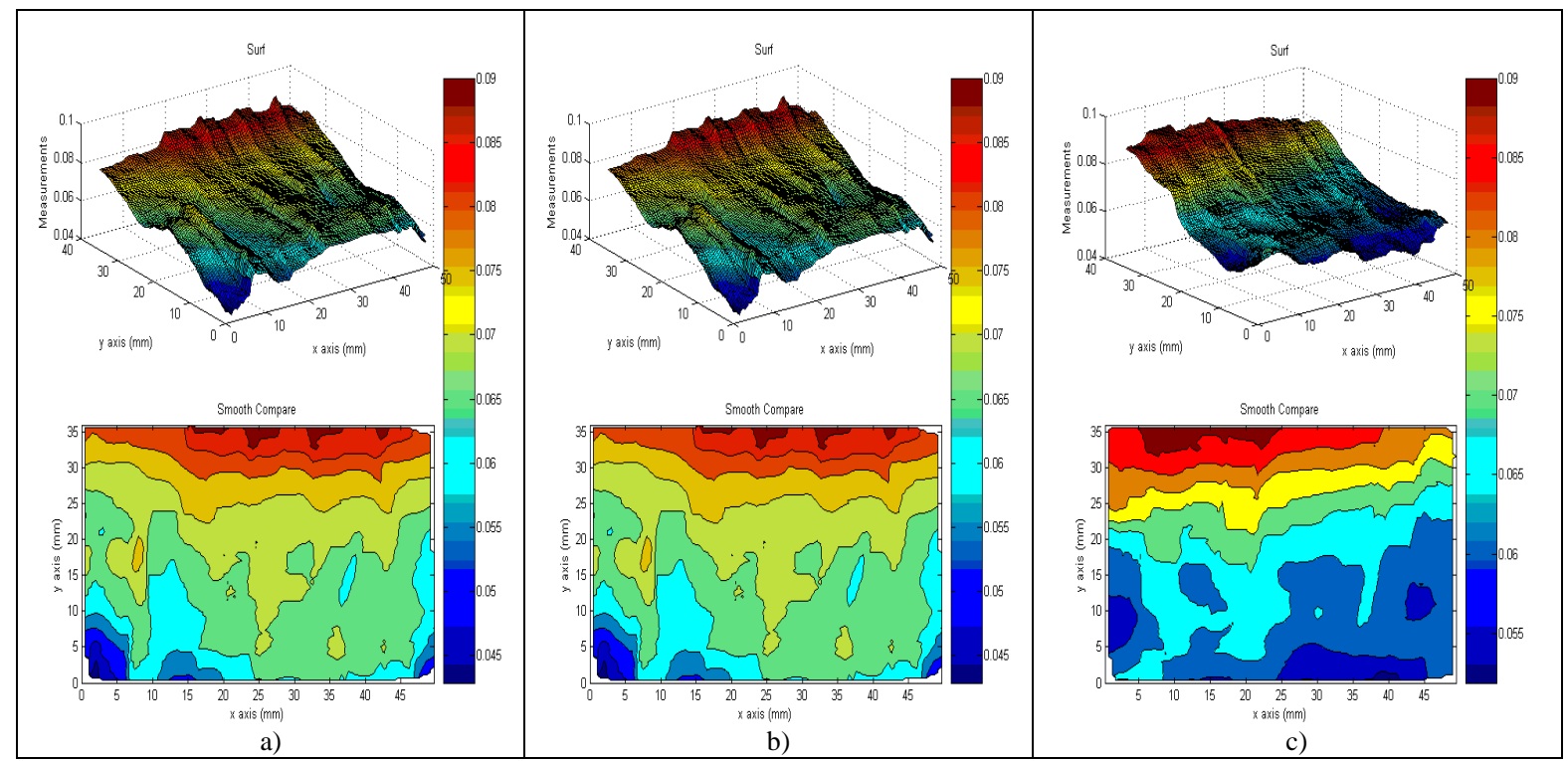

Figure 11. Comparison of strain field for samples with scarfed bonding; a) reference, b) TD1DI1, c) TD1DI2 
As it is shown the measurements represent the strain field on the scarfed bond line of the samples. In the extracted images there are some visual indications that show minor differences between the reference samples and the contaminated but no clear visual evidence was possible. So an additional statistical analysis of the extracted data was performed. The results from the statistical analysis are presented in Figure 12.

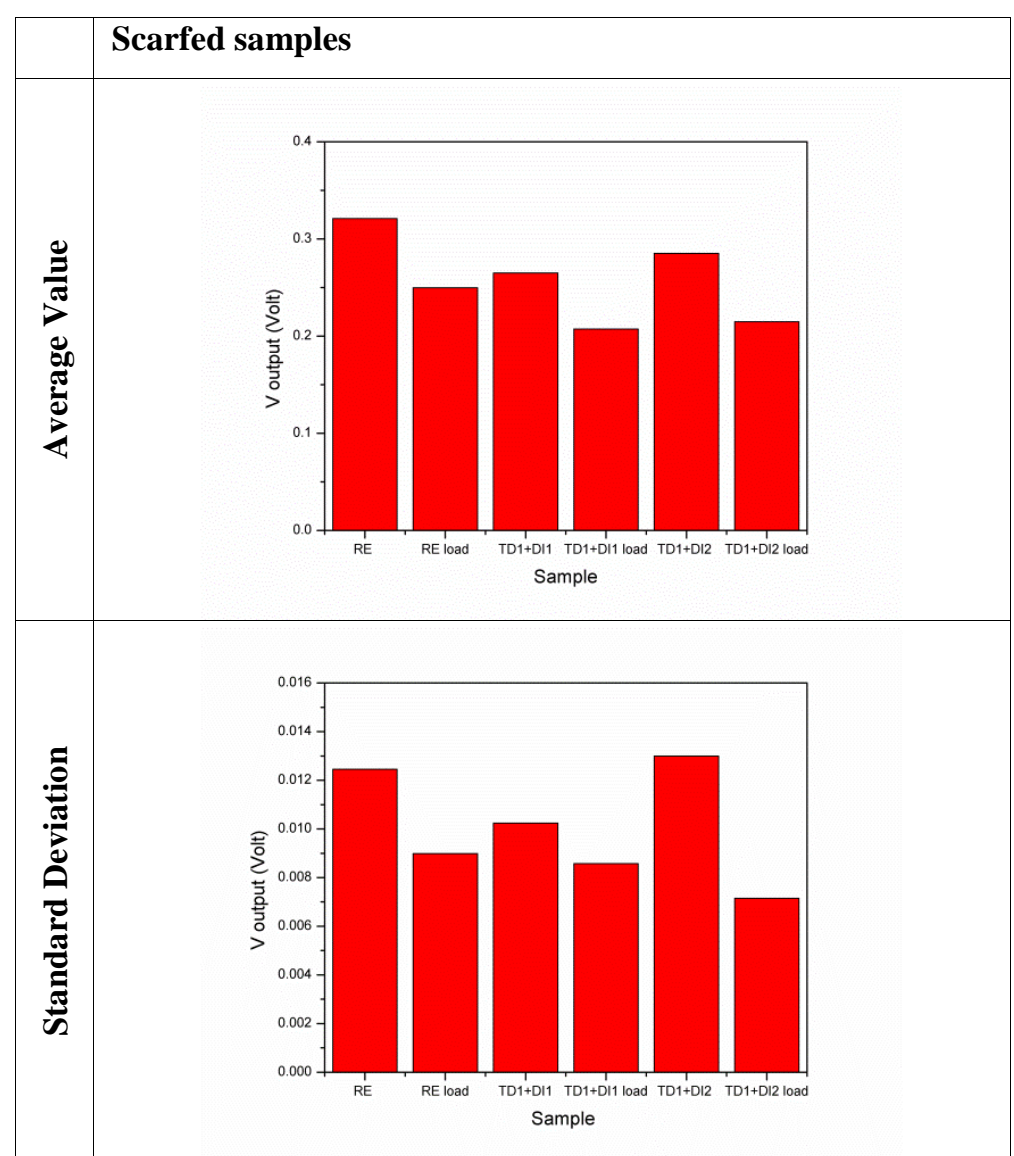

Figure 12. The average value and the standard deviation of the measurements obtained by the transducer's output at the samples with scarfed bonding 


\subsection{Laser shock adhesion test (LASAT)}

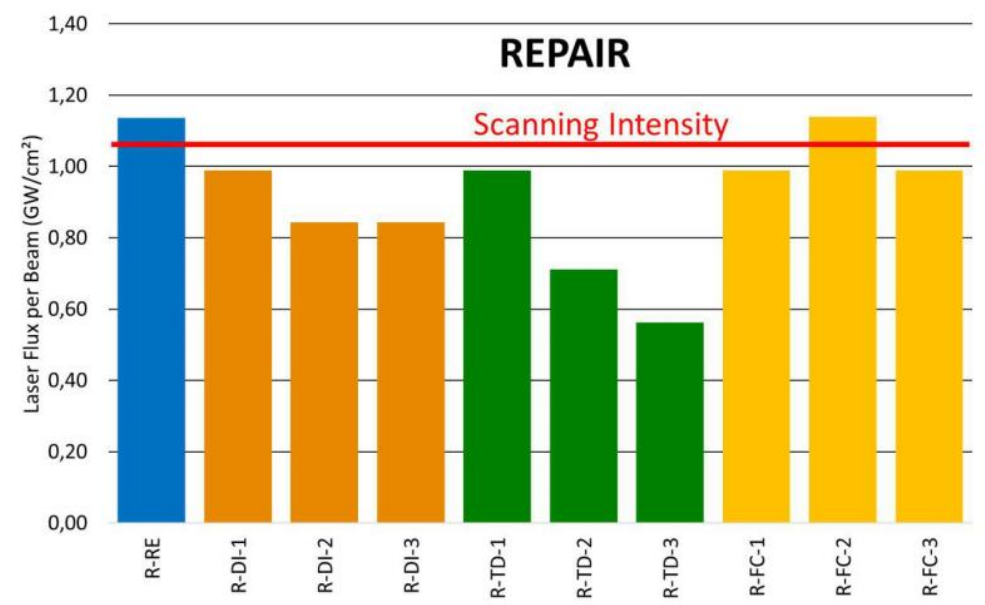

Figure 13. Summary of the results obtained for samples with single modifications

The investigated samples were divided in 16 equal areas, however, to avoid inhomogeneities, only the four center areas were tested. To determine the sample bond threshold, a series of shots has been performed on the same area, with an increasing laser intensity. After each shot, the area was scanned using ultrasounds, if no default was spotted, the laser energy would be increase, until a damage was detected. The intensity increase between two shots has been set at $10 \%$ of the maximum intensity achievable by the facility. Laser power density stability is $2 \%$ over the test. Results are presented in Figure 13. The graph shows, the laser power density required to open the bond. The reference sample is represented in blue. In order to differentiate a sound specimen from a contaminated one, their thresholds have to be different. Moreover, for the LASAT to be used, the threshold of a contaminated sample has to be lower than the standard. If both criterions are met by a sample, it can be deduced that, in this case, the LASAT is effective. It can be seen (Figure 13) that all samples except one have lower threshold that the reference. Results for both the de-icer (DI) and the thermal degradation (TD) are consistent. A drop in the adhesion level was noticed with increasing level of bond modification.

In the next step the mixed modifications were investigated. The result is depicted in Figure 14. The increase of contamination is not indicated as weak bond. The homogeneity of the contamination can be one of the reason leading to this output. These results are still under investigation, and further tests need to be performed before any conclusion can be drown.

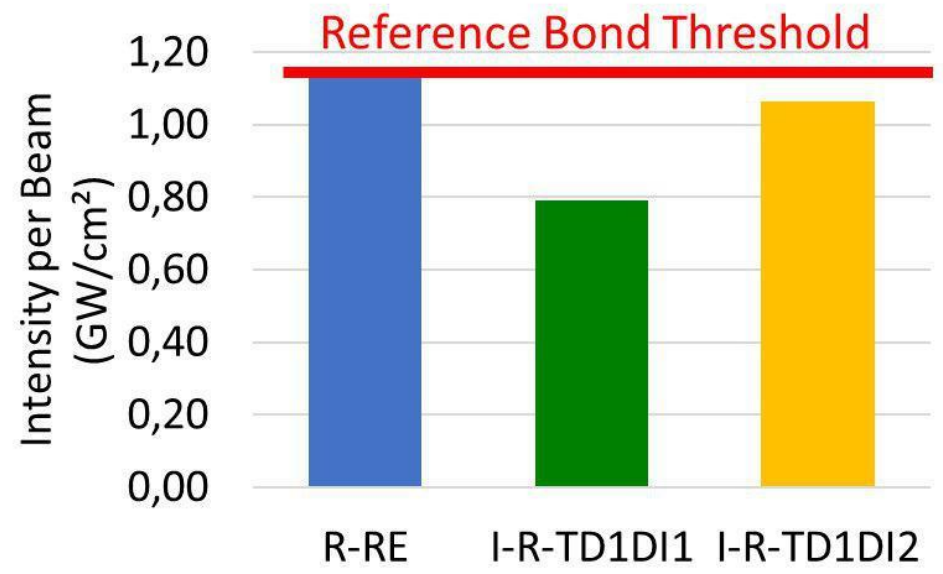

Figure 14. Summary of the results obtained for samples with mixed modifications 
The last test involved the samples with scarfed bonding. By definition, a scarfed sample has different depths of bonding, depending on the area tested. The symmetrical laser shot can easily adapt to this kind of configuration. Results are summarized in Figure 15. For this type of geometry and contamination, the LASAT could clearly differentiate a sound sample from a contaminated one. The scarfing did not affect the results, and the LASAT showed a good behaviour toward scarfed samples.

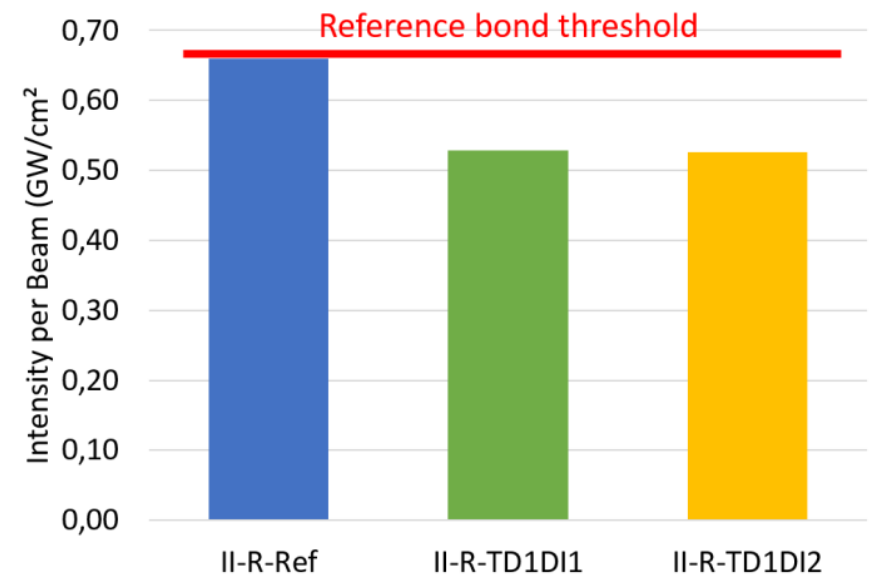

Figure 15. Summary of the results obtained for the scarfed samples

\subsection{Electromechanical impedance (EMI)}

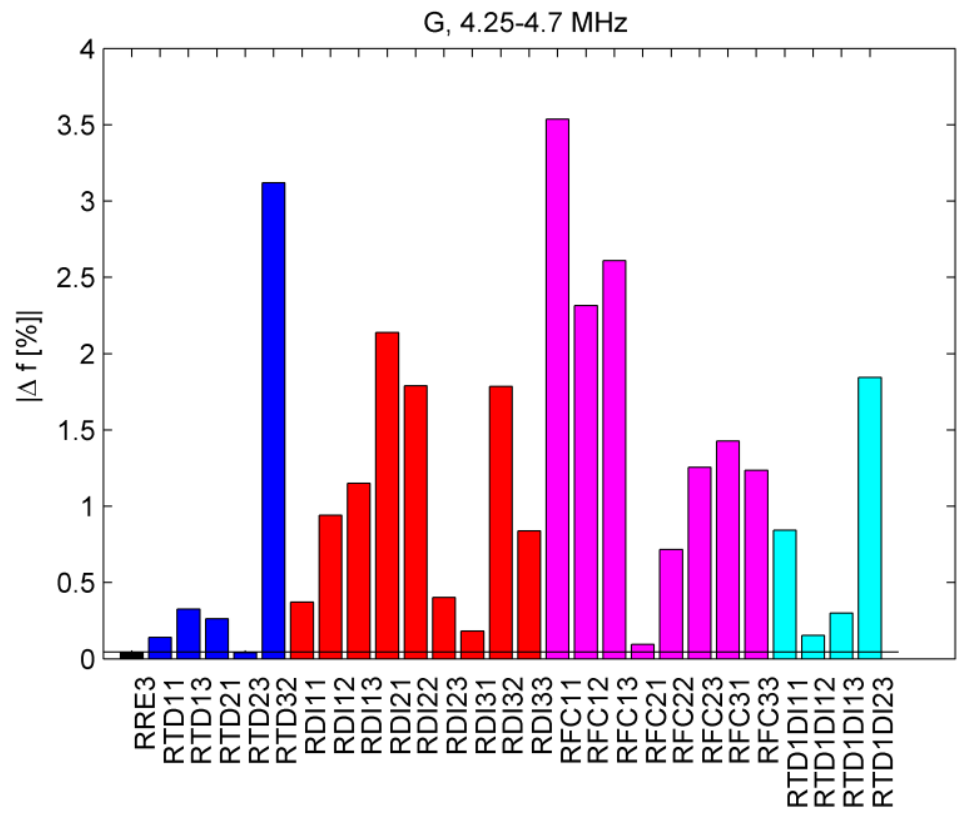

Figure 16. Conductance peak shift in the $4.25-4.70 \mathrm{MHz}$ range

In the first step the flat samples with single and mixed contamination were investigated. After looking at the registered spectra some of the results were rejected (RRE2, RTD12, RTD22, RTD33, RFC32, RTD1DI21, RTD1DI22) due to 
different shapes of the spectra. Moreover, previous results showed that RTD31 sample was delaminated [9]. The spectra were inspected in $4.25-4.70 \mathrm{MHz}$ region. A local resonance was observed except for the sample with delamination. The resonance location was tracked with respect to RRE1 sample result. The relative frequency shift was plotted in Figure 16. All the samples are above the reference threshold except the RTD23. In the case of thermal treatment (TD) the increase of the treatment temperature results in the increase of the shift. In the case of de-icer (DI) contamination such relating was not observed. The most sever faulty curing was applied to FC1 samples and this was also observed at the results obtained with EMI method. The results plotted in Figure 16 show also sensitivity of the frequency shift to the mixed modification level. However these result should be treated with caution because only one sample at the higher level of modification was studied. In the case of scarfed samples the approach to the analysis was a bit different. The spectra of free sensors and the spectra after bonding these sensors to scarfed samples were inspected. The sample RTD1DI22S was rejected from the analysis due to different spectrum curve. For the rest of the samples, it was observed that the conductance curve for free sensors is low in the range: 3.95-4.15 MHz while for the scarfed samples there is a local resonance of the conductance. The changes of the magnitude of this resonance in relation to the one of the reference samples (RRE1S) were plotted in Figure 17. In comparison with reference samples, it can be noticed that magnitude is higher for all the samples with contaminated bond except for the RTD1DI12S sample. The differences were detected but it cannot be stated that the contamination levels can be distinguished.

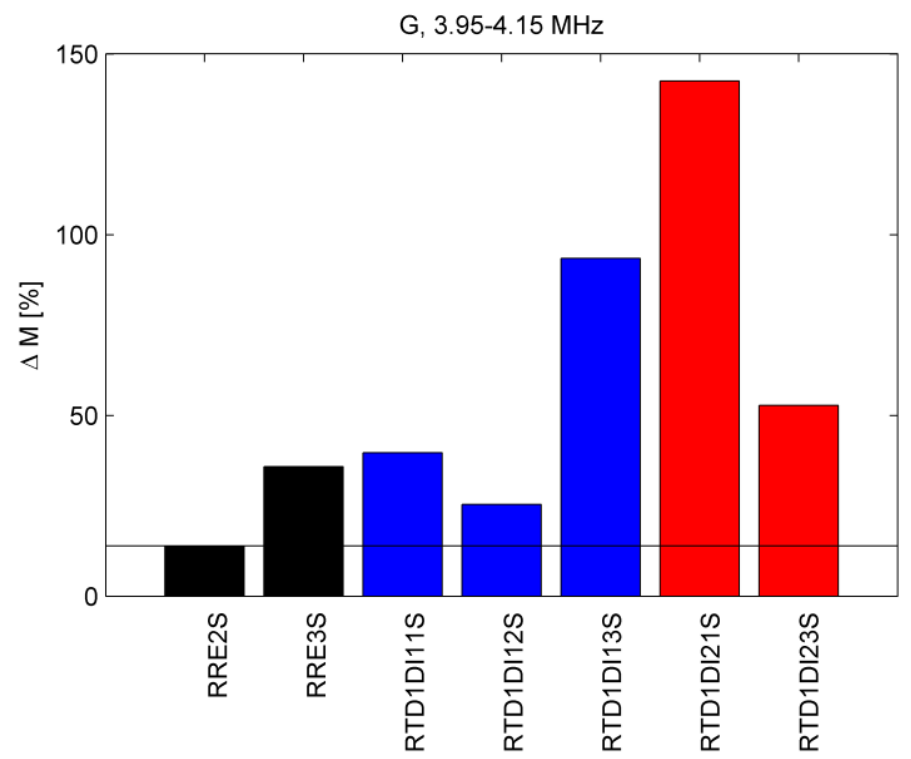

Figure 17. Change of conductance magnitude in range 3.95-4.15 MHz for the scarfed samples; the relative change was calculated in relation to the RRE1S reference sample

\section{CONCLUSIONS}

Using the $\mu$-Computed Tomography it is possible to evaluate the layer porosity of the bond lines. The observed values range from $0.3 \%$ to around $4 \%$. The single pores of the repair cases can mainly be detected along the fibres within the bond lines. The pore distribution among the three same samples of each application case is varying. This is properly because the samples were cut out of on plate with heterogeneous distribution of pores. A relation of degree of layer porosity and the different intensities (low, medium, high) as well as among the cases was not possible. This is due to the distribution of the pores. The reference samples for scarf bonding have a very high degree of porosity and is higher than of the flat reference samples. It is not possible due to the high degree of pores to distinguish among the samples of each set of different mixed contaminations.

The nonlinear investigations showed that a contaminated boundary layer of the adhesive contributes to an overall nonlinear response of the laminate that enables to recognize the difference in bonding quality caused by various types 
and levels of contamination. All kinds of single contaminations result in enhancement of the nonlinear response of the CFRP laminate which is an indication of deterioration of bonding quality. For each type of contamination, the values of $N$ change noticeably with variation of the contamination level that indicates the sensitivity to the changes in the contaminated boundary layer between the adhesive and the adherents. An anomalous increase in nonlinearity is revealed for a high level of thermal degradation that illustrates a strong loss of bonding strength induced by thermal degradation. A similar (but somewhat lower) increase in nonlinearity reveals and quantifies decrease in bonding strength caused by di-icing fluid contamination and faulty curing. Since nonlinearity enhances due to "softening" of the material, the increase in $N$ is an indication of "weakening" of the adhesive bonding. The effect of multiple contaminations confirms a cumulative decline of adhesion caused by increase of the contents of single contamination components.

The magnetostrictive strain sensing results showed detection of RTD and RFC cases. First level of mixed modification in the flat and scarfed samples were detected. all the cases of mixed contamination for both flat and curved samples were identified. The average value of the obtained data demonstrates that there is good repeatability of this method. The calculated standard deviation of the obtained data indicates that in some cases the strain field contains greater anomalies compared to the reference cases. Moreover, it was observed that in some cases after the bonding of the sensing patch there are residual stresses on the samples which induce a residual strain field. The effect of residual stresses is less noticeable when the magnetostrictive ribbons are placed parallel to the direction of the carbon fibres.

The LASAT technique generated good results for the samples with single modifications as well as the scarfed samples. It was able to correctly separate a sound sample from a contaminated one, regardless of the degree of contamination. As the results for scarfed samples indicate, the position of the bond does not matter, and the technology can be easily adapted to overcome the geometry modification. A more in-depth study still has to be done concerning the multiple contamination samples.

The EMI technique allowed for detection of almost all of the flat samples with modifications. The numerical parameter for comparison was defined after inspecting spectrum of each samples and also for free sensors. In the case of thermal treatment and faulty curing the highest level of modification was easily indicated. This was also possible for the mixed modification case. In the case of scarfed samples detection of 4 out of 5 samples was possible. The sensitivity to the modification level in not obvious. Other parameter and processing methods are also investigated for this task.

\section{ACKNOWLEDGMENTS}

This project has received funding from the European Union's Horizon 2020 research and innovation program under grant agreement no 636494 - project 'Quality assurance concepts for adhesive bonding of aircraft composite structures by advanced NDT'. Authors would like to thank teams from: University of Patras, Aernnova, Airbus Defense and Space for preparing and characterization of the samples used in this research.

\section{REFERENCES}

[1] Tserpes, K.I., Markatos, D. N., Brune, K., et al., "A detailed experimental study of the effects of pre-bond contamination with a hydraulic fluid, thermal degradation, and poor curing on fracture toughness of compositebonded joints," Journal of Adhesion Science and Technology 28(18), 1865-1880 (2014).

[2] Markatos, D. N., Tserpes, K. I., Rau, E., et al., "The effects of manufacturing-induced and in-service related bonding quality reduction on the mode-I fracture toughness of composite bonded joints for aeronautical use," Composites Part B: Engineering 45, 556-564 (2013).

[3] Moutsompegka, E., Tserpes, K.I., Polydoropoulou, P., et al., "Experimental study of the effect of pre-bond contamination with de-icing fluid and ageing on the fracture toughness of composite bonded joints," Fatigue \& Fracture of Engineering Materials \& Structures 40(10), 1581-1591 (2017).

[4] Singhal, T., Kim E., Kim T.-Y., Yang, J., "Weak bond detection in composites using highly nonlinear solitary waves," Smart Materials and Structures 26, 055011 (2017).

[5] Canal, L.P., Sarfaraz, R., Violakis, G., Botsis, J., Michaud, V., Limberger, H.G., "Monitoring strain gradients in adhesive composite joints by embedded fiber Bragg grating sensors," Composite Structures 112, 241-247 (2014).

[6] Malinowski, P., Ostachowicz, W., Brune, K., Schlag, M., "Study of electromechanical impedance changes caused by modifications of CFRP adhesive bonds," Fatigue \& Fracture of Engineering Materials \& Structures 40(10), 1592-1600 (2017). 
[7] Berthe, L., Arrigoni, M., Boustie, M., et al., "State-of-the-art laser adhesion test (LASAT)," Nondestructive Testing and Evaluation 26(3-4), 303-317 (2011).

[8] Berthe, L., Fabbro, R., Peyre, P., Bartnicki, E., "Wavelength dependent of laser shock-wave generation in the water-confinement regime," Journal of Applied Physics 85(11) 7552-7555 (1999).

[9] Malinowski, P.H., Ecault, R., Wandowski, T., Ostachowicz, W.M., „Evaluation of adhesively bonded composites by nondestructive techniques," Proceedings of SPIE - The International Society for Optical Engineering, 10170, art. no. 101700B (2017). 